\title{
Electron renormalization of sound interaction with two-level systems in superconducting metallic glasses
}

\author{
E. V. Bezuglyi, ${ }^{1}$ A. L. Gaiduk, ${ }^{1}$ V. D. Fil, ${ }^{1}$ S. Zherlitsyn, ${ }^{1,3}$ W. L. Johnson, ${ }^{2}$ \\ G. Bruls, ${ }^{3}$ B. Lüthi, ${ }^{3}$ and B. Wolf ${ }^{3}$ \\ ${ }^{1}$ B. Verkin Institute for Low Temperature Physics and Engineering, 47 Lenin Avenue, Kharkiv 310164, Ukraine \\ ${ }^{2}$ California Institute of Technology, Pasadena, California 91125 \\ ${ }^{3}$ Physikalisches Institut, Universität Frankfurt, Robert-Mayer-Straße 2-4, D60054, Frankfurt, Germany
}

(Received 3 January 2000)

\begin{abstract}
The crossing of the temperature dependences of sound velocity in the normal and the superconducting state of metallic glasses indicates renormalization of the intensity of sound interaction with two-level systems (TLS's) caused by their coupling with electrons. In this paper we examine different theoretical approaches to a quantitative description of the renormalization, using the results of a low-temperature ultrasonic investigation of $\mathrm{Zr}_{41.2} \mathrm{Ti}_{13.8} \mathrm{Cu}_{12.5} \mathrm{Ni}_{10} \mathrm{Be}_{22.5}$ amorphous alloy. It is shown that the adiabatic renormalization of the coherent tunneling amplitude can explain only part of the whole effect observed in the experiment. There exists another mechanism of the renormalization affecting only nearly symmetric TLS's, which may be associated with the effect of electron density fluctuations on the interwell potential.
\end{abstract}

\section{INTRODUCTION}

The well-known tunneling model (TM) exploits only two basic parameters for the description of low-temperature ( $T$ $\Sigma 1 \mathrm{~K}$ ) behavior of the velocity $v$ and the attenuation $\Gamma$ of sound in metallic glasses. ${ }^{1}$ The parameter $C_{0}=\bar{p} \gamma^{2} / \rho v^{2}[\bar{p}$ is the density of states of two-level systems (TLS's), $\gamma$ is the deformation potential, $\rho$ is the mass density] defines the scale of variations of $v$ and $\Gamma$ in the presence of TLS's. A parameter $\eta=n_{0} \sqrt{\overline{v_{k k^{\prime}}^{2}}}\left(n_{0}\right.$ is the density of electron states at the Fermi level, $\overline{v_{k k^{\prime}}^{2}}$ is the mean square of matrix element of the electron-TLS scattering from $k$ to $k^{\prime}$ state) determines the TLS relaxation rate due to interaction with the electron environment. According to the TM, a TLS contribution to the acoustic characteristics is determined by two additive mechanisms - the resonance and relaxation ones. Under usual experimental conditions, $\omega \ll T$, where $\omega$ is the sound frequency (we use the system of units where $\hbar=k_{B}=1$ ), the resonance contribution to the variation $\delta v(T)$ of the sound velocity is always negative and represents a straight line with the unit slope in coordinates $\delta v / C_{0} v$ vs $\ln T$. The relaxation contribution is also always negative and linear, with the slope $-1 / 2$, in the same coordinates. Thus the resulting dependence $\delta v(\ln T) / C_{0} v$ is expected to be a straight line with the slope $1 / 2$. The sound attenuation is associated mainly with the relaxation interaction and is virtually independent on $T$, whereas the resonance contribution to $\Gamma$ is small $(\sim \omega / T)$.

In superconducting glasses at low temperatures $T \ll T_{c}$, the relaxation interaction is frozen out. This makes it possible to extract purely resonance contribution and thereby to verify many of TM conclusions. Acoustic measurements in the superconducting metglasses $\mathrm{Pd}_{30} \mathrm{Zr}_{70},{ }^{2,3} \mathrm{Cu}_{30} \mathrm{Zr}_{70},{ }^{4}$ and $\left(\mathrm{Mo}_{1-x} \mathrm{Ru}_{x}\right)_{0.8} \mathrm{P}_{0.2},{ }^{5}$ carried out more than a decade ago, revealed several considerable deviations from the predictions of the TM: (i) the slope of the straight line $\delta v_{n}(\ln T) / C_{0} v$ in the normal $(n)$ phase is about $1 / 4$, whereas the canonical TM slope is $1 / 2$;

(ii) at least at high frequencies (HF), the normal-state line $v_{n}(T)$ crosses the superconducting $(s)$ line $v_{s}(T)$ at $T_{\text {cr }}$ $\ll T_{c}$. In the TM this is impossible in principle;

(iii) $v_{s}(T)$ is smaller than $v_{n}(T)$ just below $T_{c}$, as it was observed in low-frequency (LF) vibrating-reed experiments $^{2,4,5}$ and HF experiments. ${ }^{3}$ According to the original TM, the sound velocity would always increase below $T_{c}$;

(iv) the sound attenuation reveals a similar anomaly: $\Gamma_{s}(T)$ exceeds $\Gamma_{n}(T)$ within a certain temperature interval, which is about $T / T_{c} \geqslant 0.8$ in HF measurements ${ }^{3}$ and extends down to $T / T_{c} \gtrsim 0.05$ in LF experiments. ${ }^{2,4}$ By contrast, the TM predicts the attenuation to be almost independent of $T$ (with small $d \Gamma / d T>0$ ), as long as the maximum relaxation rate $\nu$ exceeds $\omega$. Thus, in LF experiments, the attenuation should be insensitive to the superconducting transition, whereas in HF measurements, $\Gamma_{s}$ should either be temperature-independent just below $T_{c}$ or decrease in metglasses with low enough $T_{c}$ (or at high enough frequencies).

It was supposed in Refs. 2 and 3 that all (or most of all) deviations from the TM are related to the electron renormalization of the parameter $C$ with respect to its bare value $C_{0}$. Although possible mechanisms of this renormalization were not discussed in Refs. 2 and 3, the qualitative consideration is rather simple. Indeed, assume that $C$ decreases due to the interaction of TLS with the electron excitations. As a result, the slope of $v_{n}(\ln T)$ decreases too. On the other hand, the bare value $C_{0}$ should be restored far below $T_{c}$. Therefore the ratio of the slopes $v_{n}(\ln T)$ and $v_{s}(\ln T)$ becomes smaller than the canonical TM value $1 / 2$. An additional assumption that the parameter $C$ grows more rapidly just below $T_{c}$ than the relaxation interaction is frozen out, leads to a simple explanation of the items (iii) and (iv). The connection between the item (ii) and the renormalization of $C$ is less ob- 
vious. Nevertheless, we will demonstrate that the intersection of $v_{n}(T)$ and $v_{s}(T)$ at $T_{\text {cr }} \ll T_{c}$ is the most convincing evidence of the reduced effective value of $C$ in the $n$ phase in comparison with the $s$ phase.

The arguments in favor of the electron renormalization hypothesis have been already presented in comparatively early theoretical works devoted to both the general problem of tunneling with dissipation and a more detailed analysis of the TLS interaction with surrounding electrons (see Refs. 6 and 7, and references therein). However, any relations, suitable for a quantitative comparison with the experiment, were not derived in these works.

A straightforward theoretical analysis of the problem of the electron renormalization of the sound-TLS interaction in metallic glasses was done by Kagan and Prokof'ev. ${ }^{8}$ It was argued that one of the reasons for the decrease in $C$ in the presence of electrons is an adiabatic renormalization of the coherent tunneling amplitude. Moreover, in order to estimate this effect, it is not necessary to introduce any additional parameter, since the renormalization $\left(C_{0}-C\right) / C_{0}=\eta^{2} / 4$ is determined by the same interaction constant $\eta$ (see also Ref. 9). Although the theory ${ }^{8}$ gives some opportunity to examine its conclusions quantitatively, such a procedure was not accomplished, probably because of lack of detailed experimental data.

In the present work we test different approaches to the quantitative analysis of the sound velocity and attenuation in metglasses, on the basis of the experimental results obtained in the superconducting amorphous $\mathrm{Zr}_{41.2} \mathrm{Ti}_{13.8} \mathrm{Cu}_{12.5} \mathrm{Ni}_{10} \mathrm{Be}_{22.5}$ alloy. It is shown that the adiabatic renormalization cannot solely explain all the experimental results, and there exists an additional mechanism of renormalization, which may be related to the fluctuation rebuilding of the interwell potential introduced by Vládar and Zawadowski. ${ }^{7}$

\section{EXPERIMENTAL RESULTS}

The alloy under investigation has a high resistance with respect to crystallization in the state of overcooled melt and remains amorphous at extremely low cooling rate $\left(<10 \mathrm{~K} \mathrm{~s}^{-1}\right) .{ }^{10}$ This makes it possible to obtain bulk homogeneous samples which perfectly suit the acoustic measurements. The ultrasonic experimental technique is described elsewhere. $^{11}$

Figure 1 shows typical temperature dependence of the velocity of the transverse sound wave in the $n$ and $s$ state of $\mathrm{Zr}_{41.2} \mathrm{Ti}_{13.8} \mathrm{Cu}_{12.5} \mathrm{Ni}_{10} \mathrm{Be}_{22.5}$. The $n$-state measurements were carried out at the magnetic field $B=1.5-2.5 \mathrm{~T}$. In accordance with the TM, the curves $v(\ln T)$ represent almost straight lines, both in the $n$ and in the deep $s$ state. The growth of $v_{s}$ below $T_{c}$ reflects freezing out of the relaxation component and agrees with the TM concept. The constant $C_{0}=(2.85 \pm 0.05) \times 10^{-5}$ was determined from the slope of $v_{s}(\ln T)$ at $T<0.3 \mathrm{~K}$.

There are also obvious deviations from the TM: the ratio of slopes in the $n$ and the $s$ phase differs from its canonical value $1 / 2$ and is close to $1 / 4$, and the curves $v(T)$ for both phases intersect at some temperature $T_{\mathrm{cr}}$. Such effects have been observed before in $\mathrm{Pd}_{30} \mathrm{Zr}_{70} .{ }^{3}$ The value of $T_{\mathrm{cr}}$ is frequency dependent; particularly, $T_{\mathrm{cr}}(62 \mathrm{MHz}) \approx 0.055 \mathrm{~K}$ and

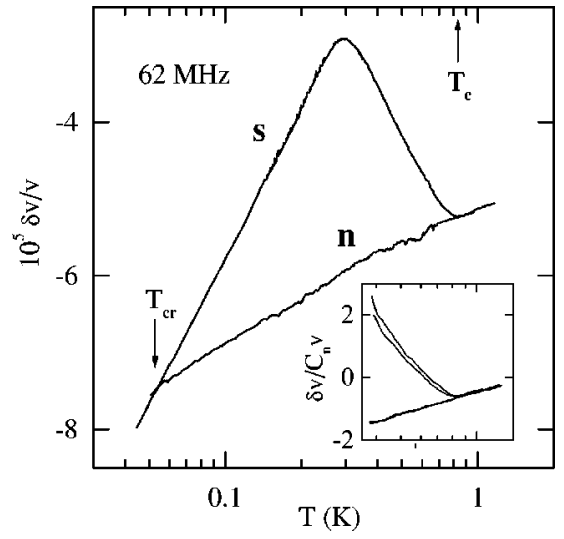

FIG. 1. Temperature variations of the transverse sound velocity in $\mathrm{Zr}_{41.2} \mathrm{Ti}_{13.8} \mathrm{Cu}_{12.5} \mathrm{Ni}_{10} \mathrm{Be}_{22.5}$ alloy in the superconducting and normal phases. Inset: normalized velocity of the transverse (upper trace, $C_{n}=6.94 \times 10^{-6}$ ) and the longitudinal mode (lower trace, $C_{n}=2.75 \times 10^{-6}$ ) near $T_{c}$. The curves were aligned in the normal phase.

$T_{\mathrm{cr}}(186 \mathrm{MHz}) \approx 0.11 \mathrm{~K}$ were found.

The inset to Fig. 1 shows the variations of $v(T)$ for the transverse $(t)$ and the longitudinal $(l)$ sound wave normalized over corresponding slopes in the $n$ phase. Obviously, these two dependencies virtually coincide, whereas relative velocity variations are essentially different.

Figure 2 shows temperature dependencies of $v_{s}$ and $v_{n}$ in the vicinity of $T_{c}$ and the diamagnetic response of the sample on the ac magnetic field $H=10^{-6} \mathrm{~T}$ at the frequency of 22 Hz. The magnetic susceptibility $\chi$ was measured simultaneously with $v_{s}$, which provides coincidence of the temperature scales for both measurements. The presence of two steps in $\chi(T)$ indicates the existence of at least two phases with different temperatures of the superconducting transition: $T_{\mathrm{cm} 1} \approx 0.9 \mathrm{~K}$ and $T_{\mathrm{cm} 2} \approx 1.0 \mathrm{~K}$. An increase of the ac field $H$ up to $10^{-5} \mathrm{~T}$ leads to complete suppression of the anomaly in the diamagnetic response at $T_{\mathrm{cm} 2}$, although the jump at $T_{\mathrm{cm} 1}$ survives up to $H=10^{-4} \mathrm{~T}$.

It is of interest to note that the temperature $T_{c} \approx 0.83 \mathrm{~K}$, at

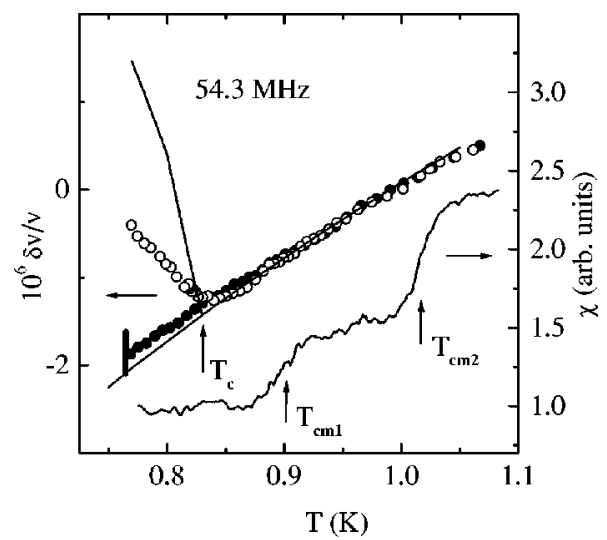

FIG. 2. Temperature variations of the magnetic susceptibility and sound velocity in $\mathrm{Zr}_{41.2} \mathrm{Ti}_{13.8} \mathrm{Cu}_{12.5} \mathrm{Ni}_{10} \mathrm{Be}_{22.5}$ in the vicinity of $T_{c}$. Open and solid circles: $B=0$ and $B=1.5 \mathrm{~T}$. Solid lines: calculations for $\eta=0.65, \varepsilon_{b}=1.2, u_{b}=0.5, R_{s}=0.14, T_{c}=0.83 \mathrm{~K}$. Thick vertical mark shows noise level. Experimental data were smoothed by adjacent averaging. 


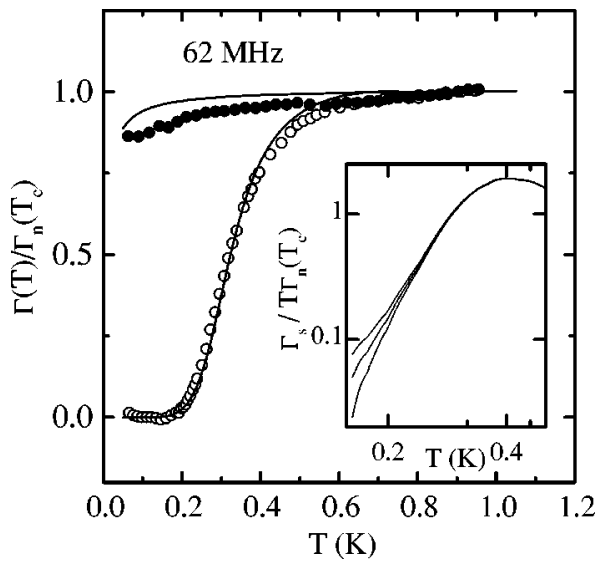

FIG. 3. Normalized attenuation of the transverse sound wave versus temperature in $\mathrm{Zr}_{41.2} \mathrm{Ti}_{13.8} \mathrm{Cu}_{12.5} \mathrm{Ni}_{10} \mathrm{Be}_{22.5}$. Open and solid circles: $B=0$ and $B=2.5$ T. Solid lines: calculated dependencies for $\eta=0.65, T_{c}=0.83 \mathrm{~K}$. Inset: to determination of the superconducting energy gap and the parameter $\eta$. The results are presented for three values of the reference level of attenuation: $-0.3 \%, 0 \%$ and $+0.3 \%$ of the total change of attenuation between the $n$ and $s$ phase, from bottom to top curve.

which we first register a nonzero difference $v_{s}-v_{n}$, coincides neither with $T_{\mathrm{cm} 1}$ nor with $T_{\mathrm{cm} 2}$. This was interpreted in Ref. 12 as a fingerprint of possible gapless superconductivity ${ }^{13}$ within the temperature region between $T_{c}$ and $T_{\mathrm{cm} 1}$. However, the magnetic scattering, which is the most prevalent reason of the gapless regime, should also reduce the superconducting energy gap when the latter opens. In our alloy, we found the energy gap to be close to the BCS value (see below), which allows us to reject such interpretation. Apparently, the diamagnetic anomalies are related to some surface phases with higher transition temperatures.

The temperature dependence of the sound attenuation is shown in Fig. 3. The experimental data are normalized on $\Gamma_{n}\left(T_{c}\right)$ which can be determined from the variations of the sound amplitude between $T_{c}$ and the deep $s$ state. The behavior of $\Gamma(T) / \Gamma\left(T_{c}\right)$ does not show any noticeable difference with similar dependencies in other superconducting amorphous alloys and reflects evolution of the relaxation contribution to the attenuation, in accordance with the TM results.

\section{QUALITATIVE CONSIDERATION}

Before making quantitative estimations, we shall discuss qualitatively a possible origin of the sound velocity anomalies in superconducting metglasses. It is naturally to associate the crossing of $v_{n}(T)$ and $v_{s}(T)$ with the enhancement of $C$ in the $s$ phase as the result of suppression of the electron renormalization at energies smaller than the superconducting gap. In order to validate this assumption, we address the expression for the resonant contribution of TLS to the sound velocity: ${ }^{14}$

$$
\left(\frac{\delta v(T)}{v}\right)_{\mathrm{res}}=P \int_{0}^{\infty} \frac{C E \tanh (E / 2 T)}{\omega^{2}-E^{2}} d E .
$$

In the simplest case of energy-independent $C=C_{0}$, in order to avoid formal logarithmic divergence of Eq. (1) at the

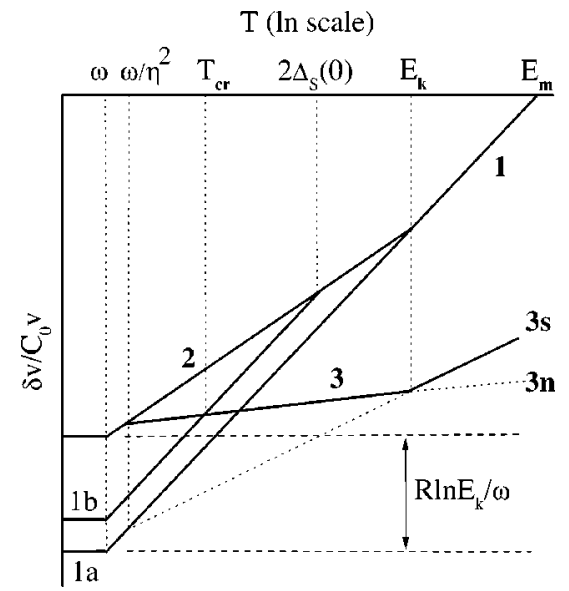

FIG. 4. Schematic representation of the temperature dependence of the sound velocity in a superconducting glass. Lines $1,1 \mathrm{a}$, and $1 \mathrm{~b}$ correspond to $\left(\delta v(T) / C_{0} v\right)_{\text {res }}$ in the superconducting phase. Line 2 represents $\left[\delta v(T) / C_{0} v\right]_{\text {res }}$ in the normal phase. Line 3 is the total $\delta v(T) / C_{0} v$ in the normal phase. See text for details.

upper limit, the velocity variations are usually referred to some arbitrary temperature $T_{0}$ :

$$
\left(\frac{v(T)-v\left(T_{0}\right)}{v\left(T_{0}\right)}\right)_{\text {res }}=C_{0} \ln \frac{T}{T_{0}}, \quad T>\omega .
$$

However, if the value of $C$ varies with energy and/or temperature, Eq. (2) is inapplicable even for qualitative estimates, since the reference value $v\left(T_{0}\right)$ may also change with $C$. To account correctly for the changes of $C$, it is necessary to analyze the whole integral in Eq. (1) by introducing a cutoff energy $E_{m}$ of the order of, e.g., melting temperature or glass transition temperature. In the case of $C=$ const, Eq. (1) can be approximated within the logarithmic accuracy by a piecewise-linear dependence (line 1 in Fig. 4):

$$
\left(\frac{\delta v(T)}{C v}\right)_{\text {res }}= \begin{cases}\ln \left(\omega / E_{m}\right), & T \leqslant \omega, \\ \ln \left(T / E_{m}\right), & T \geqslant \omega .\end{cases}
$$

Here we neglect insignificant small variations in $v$ at $T \lesssim \omega$ : a quadratic fall near $T=0$ and a shallow minimum at $\omega$ $=2.2 T$, following from the analytical solution of Eq. (1). ${ }^{15}$

Since the parameter $C_{0}$ is defined for TLS's in the absence of electron excitations, the low-temperature part of line 1 with unit slope in Fig. 4 depicts the resonance contribution to the sound velocity at temperatures much lower than $T_{c}$. Let us assume that the resonance interaction in the $n$ phase is determined by a renormalized constant $C<C_{0}$ for all energies below a certain threshold $E_{k}$. By making use of a simple steplike approximation $\left[C=C_{0}(1-R), \quad 0<R\right.$ $=$ const $<1$ for $E<E_{k}$ and $R=0$ in the opposite case], we can plot the resonance contribution in the $n$ phase by line 2 which is located over the corresponding line for the $s$ phase. Thus the resonance interaction leads to enhancement of the sound velocity in the $n$ phase at $T<E_{k}$ if $C$ decreases, and vice versa. This basic conclusion cannot be derived from Eq. (2), where "the dc component" is lost.

The relaxation contribution to the sound velocity is much less sensitive to the renormalization effects and becomes 
negligibly small at $T \lesssim \omega / \eta^{2}$. This allows us to approximate it by an expression following from Eq. (9):

$$
\left(\frac{\delta v(T)}{C_{0} v}\right)_{\text {rel }}=\frac{1}{2}\left\{\begin{array}{cc}
0, & T \leqslant \omega / \eta^{2}, \\
\ln \left(\omega / T \eta^{2}\right), & T \geqslant \omega / \eta^{2} .
\end{array}\right.
$$

The total TLS contribution to the sound velocity in the $n$ phase is schematically shown in Fig. 4 with a line 3 which was obtained in the following way. At $T<E_{k}$ the line 3 contains a piece of line $3 n$ drawn with a slope $1 / 2-R$ through the point $T=\omega / \eta^{2}$ at the line 2 . At $T>E_{k}$, the line 3 is a piece of line $3 s$ drawn with a slope $1 / 2$ from the point $T=\omega / \eta^{2}$ at the line 1 . A transition range occurs at temperatures $T \approx E_{k}$.

Let us now discuss the evolution of $v(T)$ at the superconducting transition. First, we consider a low-temperature range $T \leqq 0.3 \mathrm{~K}$. If $E_{k} \leqslant 2 \Delta_{s}(0)$ [here and below $\Delta_{s}(T)$ is the superconducting energy gap], the renormalization of $C$ should be frozen out. Thus the resonance contribution can be depicted by a low-temperature part of the line 1a. It follows from the geometry of Fig. 4, that the intersection of lines 3 and 1a is possible only at $C<C_{0}(R \neq 0)$, and the crossing temperature $T_{\mathrm{cr}}$ is given by

$(1-S) \ln T_{\mathrm{cr}}=R \ln E_{k}+(1-R-S) \ln \left(\omega / \eta^{2}\right)-A\left(T_{\mathrm{cr}}\right)$,

where $S$ is the resulting slope of $v_{n}(\ln T)$. The parameter $A$ is introduced to account for possible shift of a background level of the sound velocity in the $s$ phase with respect to the $n$ phase, normalized over $C_{0}$; it will be discussed below. According to Eq. (5), $T_{\text {cr }}$ grows along with $E_{k}, \omega$, and with the decrease in $\eta$.

At $E_{k}>2 \Delta_{s}(0)$, the renormalization of $C$ is only partially frozen out for $E<2 \Delta_{s}(0)$. Therefore the sound velocity in the deep $s$ state can be depicted as a part of the line $1 \mathrm{~b}$ (see Fig. 4). In this case, the estimate of $T_{\text {cr }}$ in Eq. (5), where $E_{k}$ is to be replaced by $2 \Delta_{s}(0)$, is also valid.

Along this line, we can also qualitatively explain the behavior of $v$ and $\Gamma$ at the superconducting transition. Below $T_{c}$, the electron renormalization rapidly reduces, and the effective $C$ grows, which leads to the decrease in $v$ and the increase in $\Gamma$. However, a competitive effect arises simultaneously: the rate $\nu$ of the TLS relaxation on electrons falls, which changes $v$ and $\Gamma$ in the opposite direction. Thus, if the phonon relaxation predominates near $T_{c}$, the effective $\nu$ varies weakly, and the sound velocity will decrease (correspondingly, the attenuation will increase) below $T_{c}$, as it was observed in Refs. 2-5. If the electron relaxation prevails (in materials with lower $T_{c}$ as in our system), the changes of $v$ and $\Gamma$ near $T_{c}$ may have any sign, depending upon the relations between $T_{c}, E_{k}$, and $\omega$.

In principle, another explanation of the crossing could be suggested. In our previous consideration, we implicitly assumed the coincidence of reference levels of the TLS contribution into the sound velocity in the $n$ and $s$ states. However, the electron subsystem itself changes the elastic properties of a metal at the superconducting transition. For example, a decrease in the electron viscosity below $T_{c}$ in pure metals leads to the change of the dislocation contribution to the sound velocity of the order of $10^{-5},{ }^{16}$ which is comparable with the TLS contribution but is undoubtedly absent in an amorphous metal. A more general mechanism is the change of the electron contribution to elastic moduli of a metal in the $s$ phase. In disordered metals with a short electron mean free path, this change is usually small $\left(\sim 10^{-6}\right)$ but in certain cases, for instance, in $A-15$ compounds close to structural instability, it may approach much larger values $\sim 10^{-4}$ of arbitrary sign. ${ }^{17}$ If we accept such a scale for the decrease in the electron contribution $A(T)$ in the $s$ state of our sample, the crossing will arise without any renormalization effects. In this case, the anomalous slope ratio could be associated with the enhancement of the density of states of asymmetric TLS which play the principal role in the relaxation interaction. Although the latter assumption contradicts the basic TM postulate about constancy of $\bar{p}$ within a wide range of tunnel parameters, we cannot reject the discussed alternative without additional argumentation presented below.

Thermodynamic treatment ${ }^{17}$ shows that the electron contribution variations in the $s$ state are independent on the sound frequency and produce a jump in the derivatives $d v_{i} / d T$ at $T=T_{c}$ proportional to $\partial^{2} T_{c} / \partial e_{i}^{2}$, for both the longitudinal $\left(e_{l}\right)$ and the transverse $\left(e_{t}\right)$ deformations. A small jump of $v_{l}$ itself is also expected at $T=T_{c}$. As the temperature decreases, the electron contribution changes as the density of a superfluid condensate, and therefore its variations become negligibly small at $T \ll T_{c}$. By making use of Eq. (5) at $R=0$ and the measured values of $S \approx 0.28, C_{0}, \eta \approx 0.65$, and $T_{\mathrm{cr}}(62 \mathrm{MHz})$, the hypothetic shift of the sound velocity between the $n$ and the deep $s$ state can be estimated as $\delta v / v \sim 5 \times 10^{-5}$. This value is comparable with the total velocity change $3 \times 10^{-5}$ for $t$ mode between $T_{c}$ and the maximum in $v(T)$ (see Fig. 1), i.e., the electron and the TLS contributions appear to be of the same order but have opposite signs. However, since the normalized TLS contribution is independent on the polarization, the data presented in the inset of Fig. 1 show that the normalized electron contribution should have the same property, i.e., the condition $\left(1 / \gamma_{l}^{2}\right) \partial^{2} T_{c} / \partial e_{l}^{2} \approx\left(1 / \gamma_{t}^{2}\right) \partial^{2} T_{c} / \partial e_{t}^{2}$ must be satisfied. The latter does not follow from the theory and can be only a result of random coincidence, which is hardly possible. Thus we conclude that the scale of temperature variations of the TLS contribution much exceeds the thermodynamic electron corrections. Furthermore, since the velocity shift $A$ is frequency independent, the following formula derived from Eq. (5),

$$
(1-S) \ln \frac{T_{\mathrm{cr}}\left(\omega_{1}\right)}{T_{\mathrm{cr}}\left(\omega_{2}\right)}=(1-R-S) \ln \frac{\omega_{1}}{\omega_{2}},
$$

shows that in the absence of renormalization $(R=0), T_{\text {cr }}$ must be proportional to $\omega$, which contradicts our experimental data. In that way, the absence of such proportionality is the most clear evidence of renormalization of the parameter $C$, irrespectively of whether a certain additional sound velocity shift between $n$ and $s$ phases exists or not.

\section{SOME RESULTS OF THE TUNNELING MODEL}

In this section we present a brief overview of the basic results of the TM used in our numerical calculations, which describe the behavior of $v(T)$ and $\Gamma(T)$ in glasses with account for the dependence of $C$ on tunnel parameters. We also discuss modifications introduced into given relations for a 
more exact account for the TLS-electron coupling., 8

The tunnel model postulates the existence of double well potentials in glasses with the tunnel coupling between wells. The density of states of TLS is constant in the space of parameters $\xi, \ln \Delta_{0}$, where $\xi$ is the potential asymmetry and $\Delta_{0}$ is an amplitude of the coherent tunneling. The response of the TLS ensemble on an external field represents an average over $\xi$ and $\ln \Delta_{0}$, which is usually performed in variables $E=\sqrt{\xi^{2}+\Delta_{0}^{2}}$ and $u=\Delta_{0} / E$. In these variables, the density of states is independent on $E$ :

$$
g(E, u)=\frac{\bar{p}}{u \sqrt{1-u^{2}}} \equiv g(u),
$$

and the TLS contributions to the sound velocity and attenuation are given by ${ }^{1}$

$$
\begin{aligned}
\left(\frac{\delta v(T)}{v}\right)_{\mathrm{res}}= & -\int_{0}^{E_{m} / T} \tanh \left(\frac{\varepsilon}{2}\right) \frac{d \varepsilon}{\varepsilon} \\
& \times \int_{0}^{1} C(\varepsilon, u) g(u) u^{2} d u(\omega \ll T), \\
\left(\frac{\delta v(T)}{v}\right)_{\mathrm{rel}}= & -\frac{1}{2} \int_{0}^{E_{m} / T} \frac{d \varepsilon}{\cosh ^{2}(\varepsilon / 2)} \\
& \times \int_{0}^{1} C(\varepsilon, u) g(u)\left(1-u^{2}\right) \frac{\nu^{2} d u}{\omega^{2}+\nu^{2}} \\
\left(\frac{\Gamma v}{\omega}\right)_{\mathrm{rel}}= & \int_{0}^{E_{m} / T} \frac{d \varepsilon}{\cosh ^{2}(\varepsilon / 2)} \\
& \times \int_{0}^{1} C(\varepsilon, u) g(u)\left(1-u^{2}\right) \frac{\omega \nu d u}{\omega^{2}+\nu^{2}} .
\end{aligned}
$$

In Eqs. (8)-(10) we introduced $\varepsilon=E / T$.

In general case, the relaxation rate $\nu$ is determined by both the electrons and phonons, but at $T \lesssim 1 \mathrm{~K}$, the phonon contribution can be neglected. In the original TM, the TLSelectron interaction is considered within a perturbation theory ${ }^{1}$ over the parameter $\eta^{2}$, which does not affect the splitting of energy levels, and all specific features of the metglass, compared with amorphous dielectrics, reduce only to the appearance of a new relaxation channel having the rate

$$
\nu=\frac{\pi \eta^{2}}{2} u^{2} T J(\varepsilon) .
$$

In the $n$ phase $J(\varepsilon)=J_{n}(\varepsilon)=(\varepsilon / 2) \operatorname{coth}(\varepsilon / 2), \nu \approx \eta^{2} T u^{2}$, and the relaxation interaction is essential for all $T>\omega$. In the $s$ state, it is necessary to use a function ${ }^{18}$

$$
\begin{aligned}
J_{s}(\varepsilon, \Delta)= & \frac{1}{2} \int_{\Delta}^{\infty} d \varepsilon^{\prime} \frac{f\left(-\varepsilon^{\prime}\right)}{\sqrt{\varepsilon^{\prime 2}-\Delta^{2}}}\left\{\frac{\varepsilon^{\prime}\left(\varepsilon^{\prime}-\varepsilon\right)-\Delta^{2}}{\sqrt{\left(\varepsilon^{\prime}-\varepsilon\right)^{2}-\Delta^{2}}}\right. \\
& \times \frac{f\left(\varepsilon^{\prime}-\varepsilon\right)}{f(-\varepsilon)} \Theta\left[\left(\varepsilon^{\prime}-\varepsilon\right)^{2}-\Delta^{2}\right] \\
& \left.\times \operatorname{sgn}\left(\varepsilon^{\prime}-\varepsilon\right)+(\varepsilon \rightarrow-\varepsilon)\right\},
\end{aligned}
$$

where $f(x)=[1+\exp (x)]^{-1}$ is the Fermi function, $\Theta(x)$ is the step Heaviside function, and $\Delta=\Delta_{s}(T) / T$. This integral coincides with $J_{n}(\varepsilon)$ for $\varepsilon \gg 2 \Delta$, it has a discontinuity at $\varepsilon$ $=2 \Delta$, and approaches $2 f(\Delta)$ for $\varepsilon \ll 2 \Delta$. A rapid decrease in $J_{s}$ below $T_{c}$ leads to freezing out of the relaxation interaction, when the maximum relaxation rate (at $u=1$ ) becomes smaller than $\omega$.

A more complicated picture was revealed beyond the perturbation theory. ${ }^{8,9}$ At $T=0$ in the $n$ phase, the bare amplitude $\Delta_{0}$ of the coherent tunneling is renormalized due to an adiabatic part of the interaction between the TLS and electrons:

$$
\Delta_{0}^{*} \propto \Delta_{0}\left(\frac{\Delta_{0}}{\omega_{0}}\right)^{\frac{\eta^{2}}{4-\eta^{2}}},
$$

where $\omega_{0}$ is of the order of the Debye energy.

In the $n$ phase at $T \neq 0$, the TLS ensemble can be divided into three energy intervals: ${ }^{9}$

1. $T \lesssim E^{*}=\sqrt{\xi^{2}+\Delta_{0}^{* 2}}$ : coherently tunneling TLS.

2. $E^{*}<T<4 \widetilde{E} / \pi \eta^{2}$ : incoherently tunneling TLS having the tunneling amplitude

$$
\tilde{\Delta} \propto \Delta_{0}\left(2 \pi T / \omega_{0}\right) \eta^{2 / 4}
$$

and the energy splitting $\widetilde{E}=\sqrt{\xi^{2}+\widetilde{\Delta}^{2}}$. In new variables $E^{*}$, $u^{*}$ and $\widetilde{E}, \tilde{u}$ within the intervals 1 and 2 , respectively, the TM relations in Eqs. (7)-(10) hold.

3. $T \gg 4 \widetilde{E} / \pi \eta^{2}$ : low-energy TLS. In this region, the amplitude of incoherent tunneling is also $\widetilde{\Delta}$ in Eq. (14), but the factor $\left(1-\tilde{u}^{2}\right)$ in Eqs. (9) and (10), which reflects the decrease in the contribution of the symmetric TLS to the relaxation interaction, is absent. Indeed, the incoherent transitions, which change the TLS energy and provide relaxation, involve even exactly symmetric TLS's, due to broadening of their energy levels. ${ }^{8,9}$ The corresponding relaxation frequency varies as

$$
\nu_{3} \propto \frac{2}{\pi \eta^{2}} T \tilde{u}^{2} \frac{\widetilde{\varepsilon}^{2}}{J(\widetilde{\varepsilon})} .
$$

One might assume that the contribution of TLS with $\widetilde{E}$ $<\sqrt{\omega T}$ should decrease due to the fall of $\nu_{3}$ at small $\tilde{\varepsilon}$. However, a numerical analysis shows that this effect is compensated by the growth of the contribution of low-energy symmetric TLS discussed above. As the result, partial contribution to $\Gamma$ and $(\delta v / v)_{\text {rel }}$ of the interval 3 virtually does not change in comparison with the original TM. A similar 
conclusion is valid for the incoherently tunneling TLS from the interval 2, since the bare density of states, Eq. (7), holds in new variables $\widetilde{E}, \tilde{u}$ due to linearity of the spectrum transformation Eq. (14) with respect to the tunneling amplitude. Thus only the contribution of the coherently tunneling TLS, undergoing the adiabatic renormalization, experiences an essential change. Indeed, the TLS density of states $g\left(u^{*}\right)$, along with the parameter $C$, acquires an additional factor $\left(1-\eta^{2} / 4\right)$ under transformation to the variables $E^{*}, u^{*}$, due to a nonlinear relationship Eq. (13) between $\Delta_{0}^{*}$ and $\Delta_{0}$. The latter rapidly changes at the superconducting transition to the linear dependence similar to Eq. (14), where $T$ is substituted for by $\Delta_{s}(T)$, and all the TM results are restored. ${ }^{8}$

\section{ANALYSIS OF THE EXPERIMENTAL DATA}

\section{A. Determination of $\Delta_{s}(0)$ and $\eta$}

Within the range of frequencies used in our experiments, rapid freezing out of the relaxation interaction begins at the temperature well below $T_{c}$ (see Fig. 3). In this case, the renormalization of $C$ is also frozen out, and the sound attenuation $\Gamma_{s}$ is described by Eq. (10), with $C=C_{0}$. According to Eqs. (10)-(12), the low-temperature part of $\Gamma_{s}(T)$ should be a straight line in coordinates $\ln \left[\Gamma_{s}(T) / T\right]$ versus $T^{-1}$ :

$$
\frac{\Gamma_{s}(T)}{\Gamma_{n}\left(T_{c}\right)}=\frac{2 \pi \eta^{2}}{3 \omega} T e^{-\Delta_{s}(0) / T} .
$$

This allows us to use the sound attenuation for a simple evaluation of $\Delta_{s}(0)$ and $\eta$ from its low-temperature dependence plotted in the inset to Fig. 3. Since this construction is very sensitive to the reference level of the attenuation, we also present two additional curves for the level variations of $\pm 0.3 \%$ of the total signal change between the $n$ and $s$ state. Within this range of the level variations, there exists a rather large temperature interval, where each curve can be well approximated by a straight line whose slope determines $\Delta_{s}(0)=1.45 \pm 0.05 \mathrm{~K}$. If we accept $T_{c}=0.83 \mathrm{~K}$, this value agrees well with the BCS relation $\Delta_{s}(0) / T_{c}=1.76$, which was used in all further calculations.

The value of $\eta=0.55 \pm 0.15$, determined by the crossing of the approximating straight lines with the ordinate axis in the inset to Fig. 3, reveals a large spread due to exponential dependence of $\eta$ on the position of the crossing point. A more accurate estimate of $\eta$ can be obtained from a numerical analysis of the attenuation within the whole temperature region of Fig. 3. By matching the most sharp part of $\Gamma_{s}(T)$, calculated from Eq. (10) at $T_{c}=0.83 \mathrm{~K}$, with the experimental dependence, we found $\eta=0.65 \pm 0.05$, in agreement with previous rough estimate.

\section{B. Sound attenuation near $T_{c}$}

The analysis performed above shows that the temperature dependence of the sound attenuation in $\mathrm{Zr}_{41.2} \mathrm{Ti}_{13.8} \mathrm{Cu}_{12.5} \mathrm{Ni}_{10} \mathrm{Be}_{22.5}$ can be rather well described by the original TM. However, within some temperature range just below $T_{c}$, the behavior of $\Gamma_{s}$ reveals anomalies which find no explanation in the original TM.

Figure 5 shows variation of $\Gamma(T)$ in the vicinity of $T_{c}$, obtained with higher resolution than in Fig. 3. The basic

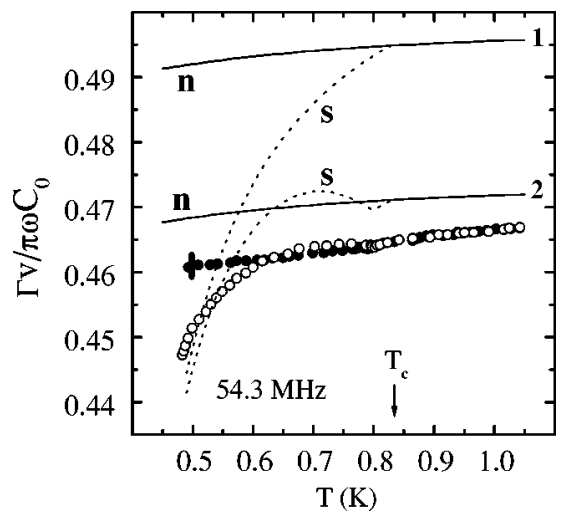

FIG. 5. Comparison of the sound attenuation near $T_{c}$ at $54 \mathrm{MHz}$ with model calculations. Open and solid circles: $B=0$ and $B=1.5$ T. Lines: related calculation for the original TM (set 1 of lines) and the TM with account for the adiabatic renormalization of the coherent tunneling amplitude, $\eta=0.65, \varepsilon_{b}=1.2$ (set 2). Thick vertical mark shows noise level, data were smoothed by adjacent averaging.

peculiarities of $\Gamma_{s}(T)$ can be discovered by a comparison of the experimental curves with the results of the original TM, Eq. (10) (line set 1 in Fig. 5). According to the calculation, a fall in the attenuation begins just at $T_{c}$ with growing slope at low temperatures. The experimental dependence has quite different behavior: $\Gamma_{s}(T)$ does not vary at $T_{c}$ within the experimental resolution and even tends to exceed $\Gamma_{n}(T)$ at lower temperatures. A more prominent excess of $\Gamma_{s}(T)$ over $\Gamma_{n}(T)$, starting just at $T_{c}$, has been previously observed in $\mathrm{Pd}_{30} \mathrm{Zr}_{70}$ alloy.

The proximity of $\Delta_{s}(0) / T_{c}$ to the BCS value shows that the explanation of these anomalies by the magnetic depairing ${ }^{12}$ is irrelevant. Let us now discuss the applicability of the concept of the electron renormalization of $C$ to the description of the attenuation.

First of all, we note that the decrease in $C$ shifts $\Gamma_{n}(T)$ downwards with respect to the original TM value $\approx 1 / 2$, for the scale used in Fig. 5. The experimental dependence actually demonstrates such a shift but, in order to analyze it quantitatively, we need the accuracy of the absolute value of the attenuation better than $1 \%$, which is beyond the possibility of our experimental technique. Therefore we shall discuss only the relative position of the curves $\Gamma_{n}(T)$ and $\Gamma_{s}(T)$ in Fig. 5, which was measured much more precisely.

As follows from Sec. IV, the most essential origin of the renormalization of $C$ is the adiabatic renormalization of tunneling amplitude, Eq. (13), which involves coherently tunneling TLS's with $E \gtrsim T{ }^{9}$. Although the thermal cutoff factor in the denominator of Eq. (10) suppresses the contribution of these TLS's, the renormalization effect remains essential on a scale of Fig. 5. In our numerical calculations, we used the following model of the energy dependence of the parameter $C$ :

$$
\frac{C}{C_{0}}=1-\frac{\eta^{2}}{4} \Theta\left(\varepsilon-\varepsilon_{b}\right)\{1+[2 f(\Delta)-1] \Theta(2 \Delta-\varepsilon)\}-B .
$$

Here $\varepsilon_{b} \sim 1$ is a fitting parameter which confines the range of the coherently tunneling TLS and reflects an exponential decrease in the coherent amplitude $\Delta_{0}^{*}$ for $\varepsilon^{*}<1 .{ }^{8}$ The last 
factor in the second term takes into account that for $\varepsilon<2 \Delta$ the contribution comes only from normal excitations. The last term in Eq. (17) corresponds to an additional renormalization due to the symmetric TLS; its origin will be discussed below. The influence of $B$ on the sound attenuation is negligibly small (we recall that the main contribution to $\Gamma$ comes from strongly asymmetric TLS's with $\nu_{\mathrm{opt}} \approx \omega$ or $u_{\mathrm{opt}} \approx \sqrt{\omega / \eta^{2} T} \ll 1$ ) and, at this stage, we assume $B=0$ for the sake of simplicity.

The results of simulation are plotted in Fig. 5 (lines 2). The difference between lines $1 \mathrm{n}$ and $2 \mathrm{n}$ manifests the adiabatic renormalization of $\Delta_{0}$ in the $n$ state. By matching the almost temperature-independent part of $\Gamma_{s}(T)$ at $\eta=0.65$ with measured curves, we obtain quite reasonable value of $\varepsilon_{b}=1.2 \pm 0.1$. The calculated dependence of $\Gamma_{s}(T)$ varies similarly to the predictions of the original TM just below $T_{c}$. Then $\Gamma_{s}(T)$ undergoes a break, accompanied by the change of the sign of $d \Gamma / d T$ at $T=2 \Delta_{s}(T) / \varepsilon_{b}$. These features arise due to exploiting a step approximation in Eq. (17), which makes the parameter $C$ insensitive to the superconducting transition until $2 \Delta_{s}(T)$ is smaller than $E=T \varepsilon_{b}$. Obviously, a smoothed energy dependence of the cutoff factor in Eq. (17), as well as possible broadening of the superconducting transition in an amorphous sample, would decrease the variation of $\Gamma_{s}(T)$ at $T_{c}$ and eliminate the break.

In this way, the evolution of $\Gamma_{s}(T)$ in the vicinity of $T_{c}$ is determined by two factors: a fall due to the decrease in the relaxation rate $\nu$, and a growth related to freezing out of the renormalization of $C$. The first factor is frequency dependent, in contrast to the second one, and the resulting variations of $\Gamma_{s}$ should also depend on frequency. When $\omega$ decreases, the temperature range, in which $\Gamma_{s}(T)>\Gamma_{n}(T)$, should be extended and vice versa. In particular, if $\eta, T_{c}$, and $\varepsilon_{b}$ are fixed, the increase in frequency by an order of magnitude (see, for example, Ref. 3, where the measurement frequency was about of $600 \mathrm{MHz}$ ) has to mask the action of the second factor completely. In this situation, $\Gamma_{s}(T)$ is always smaller than $\Gamma_{n}(T)$, and $d \Gamma_{s}(T) / d T$ grows as the temperature decreases. However, the experiments in Ref. 3 were carried out in the metglass with $T_{c}=2.6 \mathrm{~K}$, where $\nu$ is essentially determined by phonons and depends weakly on the state of the electron subsystem. Under these conditions, freezing out of the renormalization should give even stronger effect than observed in our case.

\section{Sound velocity}

The resonance contribution to $v(T)$ is completely determined by the coherently tunneling TLS with $\varepsilon \gtrsim 1$. Therefore the adiabatic renormalization of $\Delta_{0}$ in the $n$ state leads to $\left(\delta v / C_{0} v\right)_{\text {res }}=\left(1-\eta^{2} / 4\right) \ln T$ independently on the magnitude of $\varepsilon_{b}$ in Eq. (17). From Eqs. (9) and (17), we estimate the relaxation contribution as $\left(\delta v / C_{0} v\right)_{\mathrm{rel}}=-1 / 2\{1$ $\left.-\eta^{2} / 4\left[1-\tanh \left(\varepsilon_{b} / 2\right)\right]\right\} \ln T$. Using the values of $\eta$ and $\varepsilon_{b}$ obtained before, we get $\left(\delta v / C_{0} v\right)_{n}=0.42 \ln T$ for the total change in the $n$ state, whereas the slope of the experimental dependence $\left(\delta v / C_{0} v\right)_{n}=0.28 \ln T$ differs from the original TM coefficient 0.5 much more. Thus the adiabatic renormalization of $\Delta_{0}$ can solely provide less than a half of the whole effect, and therefore an additional origin of the electron renormalization of $C$ has to exist. It must affect mainly the symmetric TLS which do not participate in the relaxation attenuation. The both mechanisms can be considered as additive, because the scale of the renormalization of $C$ is small.

One of possible additional mechanisms of the renormalization is associated with the effect of electron-density fluctuations on the barrier height of the interwell potential. ${ }^{7}$ It was argued that below some critical temperature $T_{k}$, almost symmetric TLS and a surrounding electron cloud can form a strongly correlated (bound) state similar to the Kondo state. This effect has an energy threshold, $E<E_{k}\left(T_{k}\right)$. The fluctuations also lead to the renormalization of the tunnel amplitude similar to Eq. (14):

$$
\bar{\Delta}_{0}=\Delta_{0}(T / D)^{m}
$$

where $D$ is of the order of the Fermi energy. The exponent $m$ depends on $\eta$ and is about of $0.1-0.2$ for $\eta \sim 0.65$.

Formally, the spectrum transformation in Eq. (18) does not result in the renormalization of the parameter $C$, since it does not change the density of states within the space of new variables $\bar{u}, \bar{E}$. Nevertheless, the renormalizing factor in Eq. (18) would implicitly depend on $u=\Delta_{0} / E$ because, according to Ref. $7, \bar{\Delta}_{0} \rightarrow \Delta_{0}$ at $u \rightarrow 0$. This nonlinear relation between $\bar{\Delta}_{0}$ and $\Delta_{0}$ means the effective renormalization of the density of states similar to the adiabatic renormalization. One can also expect a reduced value of the deformation potential in the bound state. These aspects of the fluctuation effects were not discussed in Ref. 7.

Within such approach, the slope of $\left(\delta v / C_{0} v\right)_{\text {res }}$ is determined by the relation $C / C_{0}=1-\eta^{2} / 4-R_{s}$, where $R_{s}$ describes the contribution from the symmetric TLS in the $n$ phase. This contribution does not change the slope of ( $\left.\delta v / C_{0} v\right)_{\text {rel }}$, therefore $R_{s}$ is not a fitting parameter: at given $\eta$, its value is unambiguously determined by the resulting slope $S$ [with the account for a small contribution of adiabatic renormalization of $\Delta_{0}$ in $\left.\left(\delta v / C_{0} v\right)_{\text {rel }}\right]$. In our case, the estimates presented above give $R_{s} \approx 0.14$.

For computing, we model $B$ in Eq. (17) by simplest step function, with introducing a conditional lower boundary $u_{b}$ of the "symmetric", TLS:

$$
B=\frac{R_{s}}{\sqrt{1-u_{b}^{2}}} \Theta\left(u-u_{b}\right)\{1+[2 f(\Delta)-1] \Theta(2 \Delta-\varepsilon)\} .
$$

The meaning of the two last factors in Eq. (19) is clear from preceding discussion [see comments to Eq. (17)]. The weight $1 / \sqrt{1-u_{b}^{2}}$ provides cancellation of an additional factor in the slope renormalization $R_{s}$, which appears under the integration of $B$ over $u$ in Eq. (8). In this respect, the first factor in Eq. (19) may be interpreted as "real" renormalization of the parameter $C$ by the symmetric TLS. Note that a weak temperature dependence in Eq. (18) will introduce a correction into the slope $\left(\delta v(\ln T) / C_{0} v\right)_{\text {rel }}$. Then the value $R_{S}$ in Eq. (19) should be decreased by a factor of $(1+m)$.

A comparison between the calculated and the experimental dependencies for $u_{b}=0.5$ is presented in Fig. 6. Here we use the following procedure. The experimental points for the frequency of $62 \mathrm{MHz}$ were taken from Fig. 1 and normalized on $C_{0}$. The mutual position of $v(T)$ for different frequencies, which cannot be measured with a necessary accuracy of 


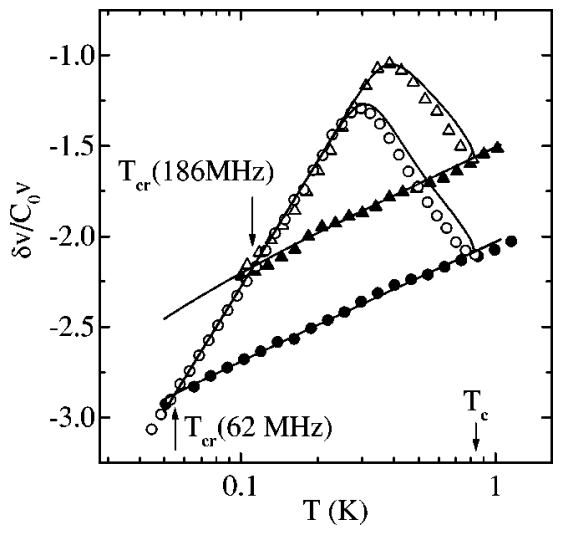

FIG. 6. Comparison of the experimental change of sound velocity for different frequencies and magnetic fields (circles: $62 \mathrm{MHz}$, triangles: $186 \mathrm{MHz}$; open symbols: $B=0$, solid symbols: $B=2.5 \mathrm{~T}$ ) with the calculations. The following set of parameters is used: $\eta$ $=0.65, \varepsilon_{b}=1.2, u_{b}=0.5, R_{s}=0.14, T_{c}=0.83 \mathrm{~K}$.

$10^{-7}$, was fixed by the alignment of their low-temperature parts. The latter are related to the resonant contribution which, according to Eq. (3), does not depend on frequency at $\omega \ll T$. The positions of the calculated curves at $v-T$ plane are determined by the upper limit $E_{m} / T$ of integration in Eqs. (8) and (9). The cutoff parameter $E_{m}$ cannot be extracted from the relative variations of the sound velocity; the only restriction, $E_{m}>10 \mathrm{~K}$, is imposed by linearity of $v_{n}(\ln T)$ at $T<1.5 \mathrm{~K}$, which is noticeably distorted at smaller $E_{m}$. At $E_{m}>10 \mathrm{~K}$, the mutual location of the calculated curves, as well as the value of $T_{\text {cr }}$, become independent on the cutoff parameter which, therefore, does not play any important role in our consideration. This allows us to use a conditional value $E_{m}=20 \mathrm{~K}$ in our calculations of the relative variations of $v$ and then to align the results with the experimental dependencies of $v_{s}$ at $T \ll T_{c}$. Under appropriate choice of $\eta$, $R_{s}$, and $\varepsilon_{b}$ discussed above, the slopes of the experimental and the calculated dependencies $v_{n}(\ln T)$ also coincide. Thus, at this stage, the only adjusting procedure consists in the choice of $u_{b}$ to approach the experimental magnitude of $T_{\text {cr }}$. As follows from Eqs. (9) and (19) at $\omega / \eta^{2} T \ll u_{b}^{2} \ll 1$, this fitting does not affect the slope of $v_{n}(\ln T)$ and is reduced to an appropriate shift of $v_{n}$ by the value $R_{s} \ln 1.36 u_{b}$, which plays the role similar to the parameter $A$ in Eq. (5). It is important to note that the single parameter $u_{b}$ allows us to adjust $T_{\text {cr }}$ for both frequencies.

Figure 6 shows a certain difference between the calculated and the measured dependencies from $T_{c}$ down to $0.4 \mathrm{~K}$ (see also Fig. 2); in particular, the calculated curve in the $s$ phase is noticeably steeper at $T \sim T_{c}$ than the experimental one. An estimate shows that a more smooth energy dependence of the adiabatic renormalization leads only to insignificant decrease in the slope of $\left(\delta v / C_{0} v\right)_{s}$ at $T \sim T_{c}$. The most probable reason for these deviations is the smearing of the superconducting transition and small thermodynamic corrections $^{17}$ to $v(T)$ in the $s$ phase. A small residual influence of phonon relaxation may also result in reduced experimental slope of $\left(\delta v / C_{0} v\right)_{s}$ near $T_{c}$ (Fig. 2).

Since the parameter $u_{b}$ is responsible for the relative position of $v_{n}(T)$ and $v_{s}(T)$, it should be sensitive to the presence of the thermodynamic corrections at given $T_{\mathrm{cr}}$ and therefore may vary within a wide range. Unfortunately, there is no clear way to separate the TLS effects from the electron contribution. Thus, if the latter is essential, the physical meaning of the parameter $u_{b}$, as it was primarily introduced in Eq. (19), becomes rather conventional. Nevertheless, this does not affect our conclusion about the presence of the additional mechanism, which renormalizes the contribution of almost symmetric TLS to the sound velocity and gives satisfactory description of $v(T)$ both in the $n$ and in the $s$ states by means of single extra parameter $u_{b}$. At the same time, thorough calculation of $\Gamma_{s}(T)$ with the additional parameter $B$ in Eq. (16) at $u_{b}=0.5$ yields practically the same dependence as shown in Fig. 5 for $B=0$, and, therefore, this mechanism does not affect the sound attenuation.

\section{CONCLUDING REMARKS}

Using the results of the acoustic measurements obtained in the superconducting glass $\mathrm{Zr}_{41.2} \mathrm{Ti}_{13.8} \mathrm{Cu}_{12.5} \mathrm{Ni}_{10} \mathrm{Be}_{22.5}$, we have carried out a quantitative analysis of different theoretical approaches to the electron renormalization of sound interaction with TLS's. A convincing evidence for the renormalization is the crossing of $v_{n}(T)$ and $v_{s}(T)$ at the temperature $T_{\mathrm{cr}} \ll T_{c}$, in combination with the absence of proportionality between $T_{\text {cr }}$ and $\omega$. By means of a simplest model renormalization of the parameter $C$ in the space of tunnel variables, it is possible to describe quantitatively the behavior of the sound velocity and attenuation exploiting the original tunnel model. It is sufficient to use the adiabatic renormalization of the coherent tunneling amplitude ${ }^{8,9}$ to fit the dependence $\Gamma_{s}(T)$ with the experiment. However, the behavior of the sound velocity can be described only with the help of an additional mechanism of the renormalization affecting only almost symmetric TLS's; this is the main conclusion following from our consideration. The additional mechanism can be presumably related to rebuilding of the interwell potential due to fluctuations, ${ }^{7}$ but this approach has not been developed enough to consider it to be incontrovertible.

The analysis carried out in this work allows us to evaluate several parameters using the experimental dependencies of $\Gamma(T)$ and $v(T)$. We emphasize that most of them are not fitting parameters in a common sense, because it is not necessary to vary their whole set simultaneously to determine each of them. We use the following sequence of the parameter evaluation. First of all, from the temperature dependence of the sound attenuation in the $s$ state we determine the energy gap $\Delta_{s}(0)$ and the interaction parameter $\eta$. Using the latter together with the experimental $\Gamma_{s}(T), v_{n}(\ln T)$, and $T_{\text {cr }}$, we evaluate the model parameters $\varepsilon_{b}, R_{s}$, and $u_{b}$ consequently, utilizing only the values which have been already found during previous steps. In this way, $\eta$ is the central parameter which determines all others. Therefore a question may occur: could an error in the determination of $\eta$ lead to essential redistribution of the contributions from different mechanisms? Under assumption that the electron contribution into $v_{s}(T)$ does not change below $T_{c}$, the needed value of $u_{b} \sim 0.3$ appears to be nonrealistically small already for $\eta=0.67-0.68$. On the other hand, for $\eta \leqq 0.6$ the best fit yields lower $T_{c}$. In this way, the studied alloy does not give a large choice for the variation of the parameters. 
In principle, it would be possible to do without the concept of an additional influence of electrons on the symmetric TLS and to reduce all the effects to the adiabatic renormalization of the tunnel amplitude, if we accept $\eta \approx 1$, assuming its estimate from the low-temperature "tail" of the attenuation (Sec. V A) to be unreliable. However, in this case, the overall shape of calculated $\Gamma_{S}(T)$ can be adjusted to the experimental dependence only at $T_{c}=1 \mathrm{~K}$, with noticeable deviations from the experimental data at $T \ll T_{c}$. Besides, the experimental value of $T_{\text {cr }}$ could be obtained only under assumption that in $s$ state at $T=T_{\mathrm{cr}}$ the electron contribution is reduced by $\delta v / v \approx 1.5 \times 10^{-5}$ [this estimate follows from Eq. (5)], which is comparable with the TLS contribution. Within such an approach, the resulting variations of $v(T)$ near $T_{c}$ would be determined by three equipollent mechanisms: the change of the TLS relaxation rate, freezing out of the adiabatic renormalization, and the evolution of the electron contribution. In our opinion, it is impossible to expect proper mutual compensation of their partial contributions, for both the longitudinal and the transverse modes, which could provide regular variations of $v(T)$ observed at the superconducting transition.
It is of interest to note that the parameter $E_{k}$, which appears in the fluctuation model, ${ }^{7}$ is not important in our evaluation process. Most probably, the condition $E_{k}>2 \Delta_{s}(0)$ is satisfied in the alloy under investigation, because, as follows from Eq. (5), the introduction of $E_{k}<2 \Delta_{s}(0)$ at constant $T_{\mathrm{cr}}$ should be accompanied by a decrease in $u_{b}$. Thus we believe that the parameter $E_{k}$ is optional in the description of lowtemperature features of the sound velocity and attenuation in our alloy. Apparently, the introduction of $E_{k}$ can be more fruitful for the analysis of the elastic properties of the metglass at higher temperatures.

\section{ACKNOWLEDGMENTS}

The authors thank G. Weiss for the fruitful discussion. This research was partially supported by the Ukrainian State Foundation for Fundamental Research (Grant No. 2.4/153) and the Deutsche Forschungsgemeinschaft via SFB 252. W.L.J. wishes to acknowledge the U. S. Dept. Of Energy for support under Grant No. DE-FG03-86ER45242. S.Z. would like to thank the Alexander von Humboldt Foundation for support.
${ }^{1}$ S. Hunklinger and A. K. Raychaudhuri, Progress in Low Temperature Physics, edited by D. F. Brewer (North-Holland, Amsterdam, 1986), Vol. 9.

${ }^{2}$ H. Neckel, P. Esquinazi, G. Weiss, and S. Hunklinger, Solid State Commun. 57, 151 (1986).

${ }^{3}$ P. Esquinazi, H.-M. Ritter, H. Neckel, G. Weiss, and S. Hunklinger, Z. Phys. B 64, 81 (1986).

${ }^{4}$ P. Esquinazi and J. Luzuriaga, Phys. Rev. B 37, 7819 (1988).

${ }^{5}$ F. Lichtenberg, H. Raad, W. Moor, and G. Weiss, Phonons 89, edited by S. Hunklinger, W. Ludwig, and G. Weiss (World Scientific, Singapore, 1990), p. 471.

${ }^{6}$ A. J. Leggett, S. Chakravarty, A. T. Dorsey, M. P. A. Fisher, A. Garg, and W. Zwerger, Rev. Mod. Phys. 59, 1 (1987).

${ }^{7}$ K. Vládar and A. Zawadowski, Phys. Rev. B 28, 1564 (1983); 28, 1582 (1983); 28, 1596 (1983).

${ }^{8}$ Yu. Kagan and N. V. Prokof'ev, Solid State Commun. 65, 1385 (1988); Zh. Éksp. Teor. Fiz. 97, 1698 (1990) [Sov. Phys. JETP 70, 957 (1990)].

${ }^{9}$ J. Stockburger, U. Weiss, and R. Görlich, Z. Phys. B 84, 457
(1991).

${ }^{10}$ A. Pecker and W. L. Johnson, Appl. Phys. Lett. 63, 2342 (1993).

${ }^{11}$ B. Lüthi, G. Bruls, P. Thalmeier, B. Wolf, D. Feinsterbusch, and L. Kouroudis, J. Low Temp. Phys. 95, 257 (1994).

${ }^{12}$ A. L. Gaiduk, E. V. Bezuglyi, V. D. Fil, and W. L. Johnson, Low Temp. Phys. 23, 857 (1997).

${ }^{13}$ A. A. Abrikosov and L. P. Gor'kov, Zh. Éksp. Teor. Fiz. 39, 1781 (1960) [Sov. Phys. JETP 12, 1243 (1960)].

${ }^{14}$ L. Piché, R. Mayuard, S. Hunklinger, and J. Jäckle, Phys. Rev. Lett. 32, 1426 (1974).

${ }^{15}$ S. Hunklinger and W. Arnold, Physical Acoustics, edited by W. P. Mason and R. N. Thurston (Academic, New York, 1976), Vol. 12, p. 153.

${ }^{16}$ V. I. Denisenko, V. D. Fil, E. A. Masalitin, and P. A. Bezugly, Fiz. Nisk. Temp. 7, 1093 (1981) [Sov. J. Low Temp. Phys. 7, 529 (1981)].

${ }^{17}$ L. R. Testardi, Physical Acoustics (Ref. 15), Vol. 10, p. 193.

${ }^{18}$ J. L. Black and P. Fulde, Phys. Rev. Lett. 43, 453 (1979). 